Mon. Not. R. Astron. Soc. 000,1 14(2012) Printed 15 October $2018 \quad$ (MN LATEX style file v2.2)

\title{
Ole Rømer's method still on the stage. The study of two bound eclipsing binaries in quintuple system V994 Her.
}

\author{
P.Zasche $e^{1 \star}$ and R. Uhlar̆² \\ ${ }^{1}$ Astronomical Institute, Faculty of Mathematics and Physics, Charles University Prague, CZ-180 00 Praha 8, V Holešovičkách 2, \\ Czech Republic \\ ${ }^{2}$ Private Observatory, Pohoři 71, CZ-254 01 Jilové u Prahy, Czech Republic
}

Accepted ... Received ...; in original form ...

\begin{abstract}
More than three hundred years ago, Ole Rømer measured the speed of light only by observing the periodic shifting of the observed eclipse arrival times of Jupiter's moons arising from the varying Earth-Jupiter distance. The same method of measuring the periodic modulation of delays is still used in astrophysics. The ideal laboratories for this effect are eclipsing binaries. The unique system V994 Her consists of two eclipsing binaries orbiting each other. However, until now it was not certain whether these are gravitationally bound and what their orbital period is. We show that the system is in fact quintuple and the two eclipsing binaries are orbiting each other with period about 6.3 years. This analysis was made only from studying the periodic modulation of the two periods, when during the periastron passage one binary has an apparently shorter period, while the other one longer, exactly as required by a theory. Additionally, it was found that both inner eclipsing pairs orbit with slightly eccentric orbits undergoing a slow apsidal motions with a period of the order of centuries.
\end{abstract}

Key words: binaries: eclipsing - binaries: visual - stars: fundamental parameters stars: individual: V994 Her.

\section{INTRODUCTION}

Eclipsing binaries are ideal astrophysical laboratories, and ' even more than a century of their intensive photometric , and spectroscopic monitoring, they still represent the best method to determine the masses, radii and luminosities of stars. Thanks to modern ground- (and space-) based telescopes we are able to discover these objects in other galaxies and to apply the same methods as used in our Solar neighborhood. One very specific method is the analysis of their orbital periods. Using the precise times of minima (centers of the eclipses of the components), we can determine if the system's period is constant, accelerating, decelerating, or periodically alternating. If we detect a periodic shifting of the times of minima, we determine that there is an additional component in the system, which is orbiting around the barycenter with the eclipsing pair. If the system is moderately inclined to the observer, the light from the eclipsing binary needs more and less time to reach us, as it moves away and closer to the observer as it orbits the unseen component. This method is in fact the same as used in the 17th century

\footnotetext{
* E-mail: zasche@sirrah.troja.mff.cuni.cz
}

by Ole Rømer when measuring the finite speed of light using the eclipse times of Jupiter's moons, see e.g. Cohen (1940).

\section{THE SYSTEM V994 HER}

Dealing with the eclipsing binaries, we have a few advantages. First of all, there are currently thousands of eclipsing binaries known. Moreover, the time baseline of observations for some eclipsing binaries is more than a century long. Importantly, the observations are very easy to obtain, even with small telescopes.

This is the case of one very interesting eclipsing system V994 Her (HD 170314, ADS $11373 \mathrm{AB} ; V=7.00 \mathrm{mag}$ ). In 2008 it was discovered (Lee et al. 2008) that V994 Her is the first (at that time) system consisting of two eclipsing binaries. From one point on the sky we can see two different sets of eclipses, one with the period of $P_{A}=2.08$ days, while the other one has a period of $P_{B}=1.42$ days. The star was also observed with the Hipparcos satellite (Perryman et al. 1997), however the strange eclipsing behavior was missed for about 15 years. The complete study of this interesting system was made (Lee et al. 2008) also on the basis of their new spectroscopic observations, yielding a set of physical parameters of all four eclipsing components. Their study showed 
that the system consists of two pairs: $\mathrm{A}(\mathrm{B} 8 \mathrm{~V}+\mathrm{A} 0 \mathrm{~V})$, and $\mathrm{B}(\mathrm{A} 2 \mathrm{~V}+\mathrm{A} 4 \mathrm{~V})$. All components are well-detached and still located on the main sequence. Both orbits are slightly eccentric.

On the other hand, one important question arises, whether the two eclipsing components comprise one gravitationally bounded system, or the system is only an optical binary. The mutual orbital period of the two pairs can be very long and long-time monitoring is rather time consuming. The system V994 Her also contains one more distant component observed interferometrically (Mason et al. 2001), whose period was estimated of about a few thousand years. Therefore, the authors (Lee et al. 2008) speculated that the two eclipsing pairs could be identified with these two visual components. For a brief review of quadruple systems with two eclipsing binaries see Cagaš \& Peicha (2012). Currently we know only six such systems, BV + BW Dra, V994 Her, CzeV343 (Cagaš \& Peicha 2012), KIC 4247791 (Lehmann et al. 2012), TYC 3807-759-1 (Lohr et al. 2012), and OGLE LMC-ECL-16549 (Graczyk et al. 2011), see Table 1

\section{ANALYSIS}

Here we introduce an original approach of delay-time variations of both eclipsing pairs, showing that the system is in fact quintuple and the two inner pairs are orbiting around each other with much shorter period. We obtained many new observations of both pairs, as well as re-analyzed the Hipparcos and ASAS (Poimanski 2002) photometry. The individual times of minima are presented in Table A1 These data were analyzed simultaneously in a combined approach of fitting both orbits together, using a well-known method usually called "Light-time effect", or "Light-travel-time effect" (hereafter LTTE), described elsewhere, e.g. Irwin (1959) or Mayer (1990).

This method has been used for dozens of binaries in the past, however, V994 Her is the first eclipsing binary system, where the method can be applied to both binaries. The main advantage of this approach is that both eclipsing pairs serve as strictly periodic "stellar lighthouses", whose apparent period changes can be studied.

We used our new code for computing this combined approach of deriving both inner orbits (their periods, and apsidal motion), together with the long orbit of mutual motion of the two pairs. Altogether 15 parameters were fitted, using all available times-of-minima observations (for $\mathrm{A}$ and B pairs: $(25+36)$ published minima together with $(18+17)$ our new unpublished data points).

In Figure 1 we plot the O-C diagrams for A and B pairs, showing their period changes (the y-axis) with respect to the time (the $\mathrm{x}$-axis). In these plots the positive $\mathrm{y}$ values indicate that the detected signal occurs later, while the negative values indicate earlier detection than predicted from strictly periodic linear behavior. As one can see, the rapid period changes near the periastron passage are clearly visible for both pairs. Most of the parameters for the LTTE fits for A and $\mathrm{B}$ are mostly the same. The exceptions are the omega angles (the argument of periastron, $\omega_{A}=\omega_{B}+180^{\circ}$ ), and the $(O-C)$ amplitudes $\left(A_{A}\right.$ and $A_{B}$, see below). The longterm modulation arises from the apsidal motion of the inner
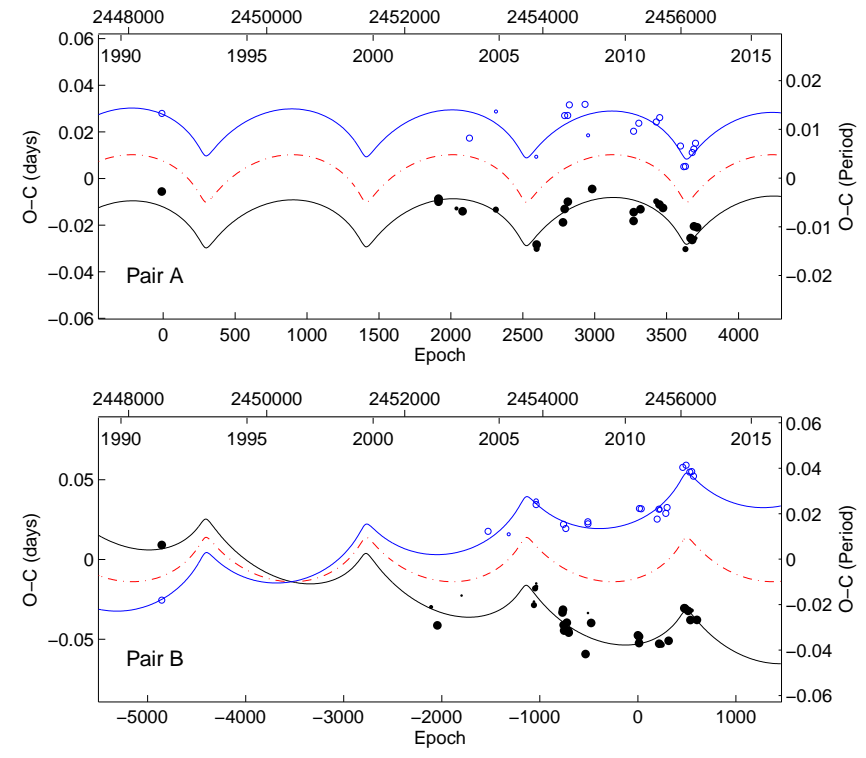

Figure 1. Plot of the O-C diagrams of both pairs. The dots stand for primary, while the open circles for the secondary minima, the bigger the symbol, the higher the weight. The red dash-dotted lines indicate the LTTE fit, while the black and blue curves represent the final fit (LTTE plus the apsidal motion).

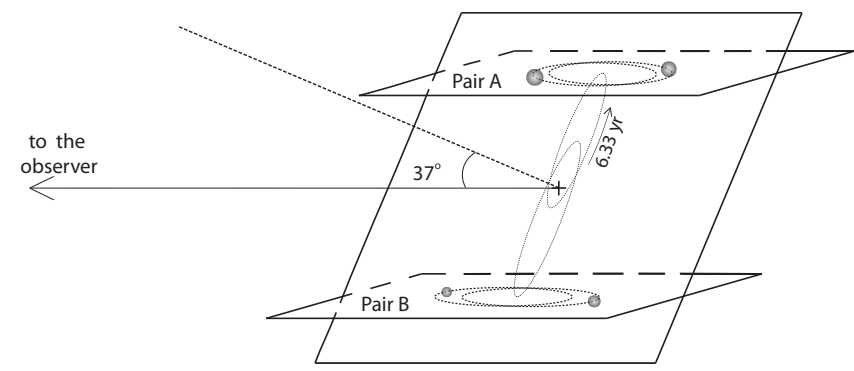

Figure 2. Schematic sketch of the V994 Her system is given (not to scale). The orbital planes of both A and B pairs are almost perpendicular to the celestial plane (i.e. edge-on to the observer, $84^{\circ}$ and $86^{\circ}$, respectively).

orbits, because both are slightly eccentric. For the final parameters of the fit see Table 2

\section{RESULTS}

The main result of our analysis is the discovery that the two eclipsing pairs orbit around each other and show also detectable period modulation together with a slow apsidal motion. The period of the motion of apsides for pair A resulted in about $(627 \pm 439)$ years, while for pair B the period is about $(113 \pm 10)$ years.

From the parameters of LTTE we determine the semimajor axis of the LTTE orbit, and using the distance from the Hipparcos satellite (Perryman et al. 1997) of $\pi=$ $(3.90 \pm 0.74)$ mas, we also derive the angular separation of the two eclipsing binaries on the sky. This resulted in an angular separation of $\Delta \alpha_{12}=(27.6 \pm 6.8)$ mas, which is well within the limits for modern stellar interferometers, hence its discovery is expect soon. Moreover, this indicates that 
Table 1. List of currently known double eclipsing systems.

\begin{tabular}{cccccccc}
\hline System Name & Other designation & RA J2000.0 & DE J2000.0 & V[mag] & Period A [d] & Period B [d] & Type \\
\hline OGLE LMC-ECL-16549 & OGLE LMC-SC3 179761 & 052809.41 & -694528.60 & 18.216 & 164.789640 & 0.818033 & EA + EW \\
CzeV343 & GSC 02405-01886 & 054824.01 & +305703.60 & 13.679 & 1.209373 & 0.806931 & EA + EA \\
TYC 3807-759-1 & GSC 03807-00759 & 093010.75 & +533859.80 & 9.538 & 1.305545 & 0.227715 & EA + EW \\
BV Dra & HIP 74370 & 151150.36 & +615125.25 & 8.040 & 0.350067 & EW + \\
BW Dra & HIP 74368 & 151150.09 & +615141.16 & 8.834 & & 0.292165 \\
V994 Her & GSC 02110-01170 & 182745.89 & +244150.66 & 7.001 & 2.083269 & 1.420038 & EA EW EA \\
KIC 4247791 & TYC 3124-1500-1 & 190839.57 & +392236.96 & 11.645 & 4.100871 & 4.049732 & EA + EA \\
\hline
\end{tabular}

Table 2. V994 Her: The final parameters of the fits for A and B pairs.

\begin{tabular}{cccccc}
\hline \multirow{2}{*}{ Parameter } & \multirow{2}{*}{ Unit } & Value & Error & Value & Error \\
\hline$J D_{0}$ & HJD & 2448501.1302 & 0.0110 & 2455375.4555 & 0.0062 \\
$P$ & Day & 2.0832691 & 0.0000038 & 1.4200381 & 0.0000051 \\
$e$ & & 0.0311 & 0.0074 & 0.1258 & 0.0032 \\
$\omega$ & Deg & 16.0 & 4.2 & 313.8 & 2.8 \\
$\mathrm{~d} \omega / \mathrm{d} t$ & Deg/Cycle & 0.0032 & 0.0013 & 0.0124 & 0.0010 \\
$p_{3}$ & Year & 6.33 & 0.56 & 6.33 & 0.56 \\
$T_{0}$ & HJD & 2456067 & 199 & 2456067 & 199 \\
$A$ & Day & 0.0102 & 0.0042 & 0.0139 & 0.0057 \\
$\omega_{3}$ & Deg & 256.2 & 24.1 & 76.2 & 24.1 \\
$e_{3}$ & & 0.747 & 0.182 & 0.747 & 0.182 \\
\hline
\end{tabular}

the previously mentioned star at $\sim 1$ arcsec away is another star, not the eclipsing one as suggested by Lee et al. (2008). Membership of this distant component to the eclipsing pairs was suggested via similar proper motions, see e.g. the WDS catalogue1, Mason et al. 2001. Thus we have a quintuple star system. We currently know only 20 quintuples (Eggleton \& Tokovinin 2008).

From the parameters of LTTE one can also calculate the mass function of the distant body

$$
f\left(m_{3}\right)=\frac{\left(m_{3} \sin i\right)^{3}}{\left(m_{1}+m_{2}+m_{3}\right)^{2}}=\frac{1}{p_{3}^{2}}\left[\frac{173.15 A}{\sqrt{1-e_{3}^{2} \cos ^{2} \omega}}\right]^{3},
$$

see e.g. Maver (1990). Using the masses of both pairs as determined by Lee et al. (2008), we can also calculate the inclination between the eclipsing binary and the LTTE orbit. If we label the inclination between the orbit of pair $\mathrm{A}$ and the LTTE orbit as " $i_{A}$ ", and vice versa for B, then one can derive:

$$
\frac{m_{A} \cdot A_{A}}{\sin i_{A}}=\frac{m_{B} \cdot A_{B}}{\sin i_{B}} .
$$

From this equation and the mass function found from the LTTE, we can directly determine the inclination between the orbits. This results in $i_{A}=37.1^{\circ} \pm 7.3^{\circ}$, while $i_{B}=$ $36.8^{\circ} \pm 6.8^{\circ}$. Evidently, the inclinations, as derived from both $\mathrm{A}$ and $\mathrm{B}$ pairs, are comparable, and hence we know the absolute orientation of the orbit in space. This is the first time that the mutual inclination between the orbits of the eclipsing pairs has been measured. Only about twenty other

\footnotetext{
1 http://ad.usno.navy.mil/wds/
}

systems with unambiguous mutual inclinations between the eclipsing and outer orbits are known, see e.g. O'Brien et al. (2011).

\section{DISCUSSION AND CONCLUSIONS}

V994 Her is an interesting target for a future study. However, there still remain some open questions. For example, these include a detection of the distant component in the spectra and determination of its orbital period, etc. Additionally, some of the orbital elements of the 6.3-yr orbit are still unknown - for instance the longitude of the ascending node $\Omega$. The long-term evolution of outer and inner orbits should be studied over longer time scales. However, detecting the mutual motion of the two eclipsing pairs is a unique discovery and we can still hope to find similar configurations also in other multiple systems.

Surprisingly, for this analysis there was no need of spectroscopic observations for the radial velocities of the longperiod system, that would be rather complicated with current observing time allocations on larger telescopes needed for such studies. It is noteworthy that all of our new observations were carried out with 20 -cm aperture or smaller telescopes by an amateur astronomer. As clearly demonstrated by this study, scientifically valuable results can be secured with small telescopes by amateur observers.

\section{ACKNOWLEDGMENTS}

The data used for the analysis are presented in the Appendix section in Table A1 We do thank the "ASAS" team 
for making all of the observations easily public available. We also thank Assoc.Prof. Marek Wolf for sending us his photometric data. Prof. Ed Guinan is also greatly acknowledged for his referee comments, which significantly improved the manuscript. This work was supported by the Czech Science Foundation grant no. P209/10/0715, by the research programme MSM0021620860 of the Czech Ministry of Education, and by the grant UNCE 12 of the Charles University in Prague. This research has made use of the Washington Double Star Catalog maintained at the U.S. Naval Observatory, the SIMBAD database, operated at CDS, Strasbourg, France, and of NASA's Astrophysics Data System Bibliographic Services.

\section{REFERENCES}

Ak, H., \& Filiz, N. 2003, IBVS, 5462, 1

Biro, I. B., Borkovits, T., Csizmadia, S., et al. 2006, IBVS, 5684,1

Biro, I. B., Borkovits, T., Hegedüs, T., et al. 2007, IBVS, 5753, 1

Borkovits, T., Biro, I. B., Hegedüs, T., et al. 2002, IBVS, 5313, 1

Borkovits, T., Biro, I. B., Csizmadia, S., et al. 2004, IBVS, 5579, 1

Borkovits, T., Biro, I. B., Hegedüs, T., et al. 2011, IBVS, 5979, 1

Brát, L., Trnka, J., Šmelcer, L., et al. 2011, OEJV, 137, 1

O'Brien, D. P., McAlister, H. A., Raghavan, D., et al. 2011, ApJ, 728, 111

Cagaš, P., \& Pejcha, O. 2012, A\&A, 544, L3

Cohen, I. B. 1940, Isis, 31, 327

Eggleton, P. P., Tokovinin, A. A. 2008, MNRAS, 389, 869

Graczyk, D., Soszyński, I., Poleski, R., et al. 2011, AcA, 61,103

Irwin, J. B. 1959, AJ, 64, 149

Lee, C.-U., Kim, S.-L., Lee, J. W., et al. 2008, MNRAS, 389,1630

Lehmann, H., Zechmeister, M., Dreizler, S., Schuh, S., \& Kanzler, R. 2012, A\&A, 541, A105

Lohr, M. E., Norton, A. J., Kolb, U. C., et al. 2012, arXiv:1210.6765

Mason, B. D., Wycoff, G. L., Hartkopf, W. I., Douglass, G. G., \& Worley, C. E. 2001, AJ, 122, 3466

Mayer, P. 1990, BAICz, 41, 231

Perryman, M. A. C., Lindegren, L., Kovalevsky, J., et al. 1997, A\&A, 323, L49

Pojmanski, G. 2002, AcA, 52, 397

Zasche, P., Uhlař, R., Kučáková, H., \& Svoboda, P. 2011, IBVS, 6007, 1 
Table A1. Heliocentric minima of V994 Her.

\begin{tabular}{|c|}
\hline HJD -2400000 \\
\hline 48480.29371 \\
\hline 48481.36885 \\
\hline 52488.4984 \\
\hline 52488.4994 \\
\hline 52488.4997 \\
\hline 52748.9041 \\
\hline 52836.4002 \\
\hline 52937.4701 \\
\hline 53319.71928 \\
\hline 53320.80295 \\
\hline 53904.09882 \\
\hline 53909.26748 \\
\hline 53909.26932 \\
\hline 54290.517 \\
\hline 54314.5204 \\
\hline 54315.522 \\
\hline 54360.3524 \\
\hline 54361.3570 \\
\hline 54383.2729 \\
\hline 54610.3494 \\
\hline 54654.08476 \\
\hline 54713.4349 \\
\hline 55313.4026 \\
\hline 55314.4827 \\
\hline 55315.4896 \\
\hline 55389.48381 \\
\hline 55413.40450 \\
\hline 55641.55985 \\
\hline 55642.56748 \\
\hline 55688.39802 \\
\hline 55691.56016 \\
\hline 55740.47826 \\
\hline 55993.62195 \\
\hline 56041.52826 \\
\hline 56064.44435 \\
\hline 56065.45050 \\
\hline 56138.36968 \\
\hline 56162.36395 \\
\hline 56163.36806 \\
\hline 56187.36470 \\
\hline 56188.37316 \\
\hline 56210.28311 \\
\hline 56211.28403 \\
\hline 56234.20460 \\
\hline 48479.75947 \\
\hline 48480.43493 \\
\hline 52471.4363 \\
\hline 52381.9854 \\
\hline 52822.20440 \\
\hline 53206.3650 \\
\hline 53504.57119 \\
\hline 53870.18880 \\
\hline 53870.18658 \\
\hline 53887.23816 \\
\hline 53887.23754 \\
\hline 53902.20258 \\
\hline 53902.20047 \\
\hline 53904.28105 \\
\hline 53904.27915 \\
\hline 54283.4131 \\
\hline 54290.515 \\
\hline 54298.3787 \\
\hline 54300.4457 \\
\hline 54307.5424 \\
\hline 54332.457 \\
\hline 54334.523 \\
\hline 54347.3084 \\
\hline 54364.3439 \\
\hline 54374.2829 \\
\hline 54618.5160 \\
\hline 54650.5498 \\
\hline 54652.62262 \\
\hline 54653.3886 \\
\hline 54699.4777 \\
\hline 55375.40806 \\
\hline 55392.44365 \\
\hline 55392.44779 \\
\hline 55397.49810 \\
\hline 55424.47871 \\
\hline 55654.51845 \\
\hline 55681.50533 \\
\hline 55683.55108 \\
\hline 55691.44530 \\
\hline 55779.48535 \\
\hline 55799.36956 \\
\hline 55821.29667 \\
\hline 56026.60079 \\
\hline 56048.52317 \\
\hline 56058.46317 \\
\hline 56073.46347 \\
\hline 56102.48296 \\
\hline 56127.42060 \\
\hline 56136.55813 \\
\hline 56154.40165 \\
\hline 56156.44467 \\
\hline 56181.37945 \\
\hline 56230.28075 \\
\hline
\end{tabular}

